\title{
Percepción social de los socios sobre las Cooperativas No Agropecuarias en Cuba
}

\author{
(Members' Social Perception of Non-Agricultural \\ Coopoeratives in (uba)
}

\author{
Deibby Valle Ríos ${ }^{1}$ \\ Universidad de Pinar del Río, Cuba \\ José Manuel Figueroa González² \\ Escuela de Administración de Empresas, España
}

Sumario: I. Introducción. II. Acerca del concepto de Percepción Social: Breve aproximación teórica. III. Cooperativas no Agropecuarias en Cuba. Especial referencia a la provincia de Pinar del Río. IV. Percepción Social de los asociados a cooperativas no agropecuarias cubanas. El caso de la CnoA Atelier «La Moda». V. Conclusiones.

Resumen. El objetivo de este trabajo es diagnosticar las principales percepciones sociales que poseen los socios acerca de su cooperativa no agropecuaria. Para ello, se fundamentan brevemente los elementos teóricos relacionados con los procesos de percepción y percepción social, y los modos en que se ha estudiado el tema desde las cooperativas. Posteriormente, se hace un recorrido por la evolución de las cooperativas no agropecuarias en Cuba y los avances de la provincia de Pinar del Río en el tema. Por último, se diagnostica la percepción social que poseen los socios de las cooperativas no agropecuarias en Cuba, haciendo especial énfasis en una cooperativa de Pinar del Río.

Palabras clave: percepción social, cooperativas no agropecuarias.

Abstract. The objective of this work is to diagnose the main social perceptions that members have about their non-agricultural cooperatives. For this, the theoretical elements related to the processes of perception and social perception and the ways in which the subject has been studied from the

1 Licenciada en Psicología por la Universidad de La Habana, Cuba (2014). Profesora del Departamento de Gestión Sociocultural para el Desarrollo. Facultad de Ciencias Sociales y Humanidades. Universidad de Pinar del Río. Cuba. E-mail: deibby@upr.edu.cu

2 Doctor en Ciencias. Profesor de la Escuela de Administración de Empresas. Barcelona. España. E-mail: jfigueroa@iceb-edu.com 
cooperatives are briefly based. Subsequently, a tour is made of the evolution of non-agricultural cooperatives in Cuba and the progress of the Pinar del Río province on the subject. Finally, diagnose the social perception that members of non-agricultural cooperatives have in Cuba, making special emphasis on a non-agricultural cooperative of Pinar del Río.

Keywords: social perception, non-agricultural cooperatives. 


\section{Introducción}

Las investigaciones desarrolladas en el campo de las ciencias sociales han contribuido a definir a la percepción y concebirla como el conjunto de procesos que garantizan en los seres humanos el reflejo de la subjetividad, formándose, corrigiéndose y comprobándose a partir de ella, las imágenes del medio externo.

Constituye el momento inicial de los procesos psíquicos, donde se modifican o seleccionan grandes cantidades de datos que nos llegan constantemente del exterior. Esta selección permite almacenar y recuperar más fácilmente de la memoria la información obtenida e ir más allá de ella, con el fin de predecir acontecimientos futuros para evitar o reducir las sorpresas.

El resultado de este procesamiento brinda a los seres humanos conocimientos inmediatos o intuitivos, además de que les permite realizar juicios o valoraciones sobre determinados hechos o personas. Cada una de estas construcciones es personal, parte de la subjetividad de los seres humanos, teniendo un papel activo al formar sus propias percepciones de la realidad. Así, somos capaces de producir la información, además de crear las condiciones en las que se genera esta.

Igualmente, percibir nos ayuda a inferir los rasgos e intenciones de otra u otras personas. A esta habilidad, los autores desde las diferentes investigaciones que han desarrollado, le han llamado percepción social. Es sí misma, la percepción social constituye un constructo psicológico, un mecanismo hipotético que se emplea para explicar ciertas relaciones entre la entrada y la salida de los estímulos y la conducta manifiesta de los seres humanos.

En Cuba, varios autores han investigado la percepción social desde diferentes puntos de vista ${ }^{3}$. Los trabajos desarrollados contribuyen a profundizar en el tema, sistematizar conceptos y obtener nuevos resultados puestos en función de la ciencia.

3 Perera. M.: Informe Final del proyecto de Investigación Percepción Social del Dirigente: Programa Nacional de Ciencia y Técnica. «El trabajo con los cuadros. (Trabajo inédito). Centro de Investigaciones Psicológicas y Sociológicas. La Habana, 2002; GoNZÁlEZ-GUIJA.: La influencia de los medios en la percepción social de la delincuencia ejercida sobre la mujer. En soporte digital. s/f; PALACIO, Y.: Identidad Nacional y Cine Cubano Contemporáneo: Una visión desde la subjetividad del espectador. Tesis en opción al grado de Máster en Psicología Social y Comunitaria. Universidad de La Habana, 2005; GÓmez, G.: «Sentirse parte. Un estudio sobre la Percepción Social de la discriminación por VIH y sida». Tesis en opción al grado de Máster en Psicología Social y Comunitaria. Facultad de Psicología. Universidad de La Habana 2009. 
En estos aportes destaca Gómez ${ }^{4}$ quien en su estudio clasifica a las percepciones sociales en coincidentes y divergentes. Sobre su criterio nos hemos apoyado para realizar nuestro trabajo, con la intención de diagnosticar esencias, ya sean comunes o no, en los socios de las cooperativas no agropecuarias cubanas (CnoA).

La revisión bibliográfica nos habla de la poca sistematización en el tema desde las ciencias sociales. Escasos trabajos describen las percepciones que de sus cooperativas poseen los asociados, ya sea en nuestro país o en contextos foráneos. Los realizados hasta la fecha, no logran una representatividad de las muestras, y en otros casos se centran en el estudio de una variable en específico, imposibilitando que se realicen diagnósticos y caracterizaciones profundas sobre el tema.

Teniendo en cuenta esta realidad, nos proponemos desarrollar una serie de trabajos que profundicen en la percepción social de los asociados sobre sus cooperativas, a fin de perfeccionarlas y contribuir a crear espacios de satisfacción y realización para ellos.

Primeramente, para lograrlo, hacemos un breve recorrido por la evolución que han tenido las cooperativas no agropecuarias en Cuba, así como las cifras que las representan en los sectores de la economía donde se han constituido.

Este segundo epígrafe dedica especial atención además a la provincia de Pinar del Río, al poseer esta una tradición histórica en la implementación de cooperativas, tanto agropecuarias - al triunfo de la Revolución-, como no agropecuarias, desde el año 2013. Su experiencia con estas empresas, además de la existencia de instituciones como la Universidad de Pinar del Río, que cuenta con el Centro de Estudios de Dirección, Desarrollo Local, Turismo y Cooperativismo (CE-GESTA), y el Departamento de Derecho de la Facultad de Ciencias Sociales y Humanidades, nos llevan a trabajar directamente con el territorio, a fin de aprovechar estos espacios para retroalimentarnos de las investigaciones realizadas hasta el momento y contar con el apoyo que pudieran brindarnos en el acceso y trabajo con la muestra.

Finalmente, en el tercer epígrafe de la investigación se declaran las principales percepciones sociales de los asociados a cooperativas no agropecuarias cubanas. El análisis parte de las percepciones más comunes diagnosticadas a nivel nacional, las encontradas en las CnoA pinareñas y más específicamente, las que poseen las socias de una cooperativa no agropecuaria que sobresale por su trayectoria destacada en la implementación de este modelo de gestión no estatal en la provincia.

4 GÓMEz. G. ob. cit., p. 95. 


\section{Acerca del concepto de Percepción Social: breve aproximación teórica}

La percepción ha sido considerada el momento inicial de los procesos psíquicos. Corrientes como el materialismo dialéctico y la teoría leninista del reflejo la consideran la imagen concreta sensorial de la realidad, primer escalón del conocimiento, sobre la cual se concibe el mundo de forma abstracta, lógica y teórica. También aparece como el eslabón inicial del procesamiento de la información, ya que es el producto de la actuación de diferentes estímulos sobre los órganos sensoriales ${ }^{5}$.

Varios autores la consideran un proceso activo, histórico y con un carácter objetal ${ }^{6}$. Desde la concepción de proceso activo, la percepción constituye el conjunto de procesos que garantizan el reflejo subjetivo, parcial y al mismo tiempo adecuado de la realidad. En ellos se forma, se corrige y se comprueba la imagen de la realidad, siendo esta construcción una condición necesaria para el éxito de cualquier actividad.

Percibir también es una forma de aprendizaje, que se ha ido adecuando a la realidad a partir de la propia interacción social. Lo anterior convierte a la percepción en un proceso tanto histórico como social.

Además, en su estructura también se define el carácter objetal de las percepciones que no es más que las atribuciones que se realizan a los propios objetos de la realidad, y no a superficies estimuladas de los órganos de los sentidos, de todas las informaciones recibidas del mundo externo.

Este carácter objetal puede manifestarse a partir de tres modos diferentes ${ }^{7}$ :

- La integridad.

- La constancia.

- La racionalidad.

El primero de estos modos permite que las personas relacionen las cualidades de los estímulos, es decir, que no les lleguen de forma aislada, y más aún, que perciban los objetos como totalidades.

5 Bello. Z.: Psicología general. Editorial Félix Varela. La Habana, 2007, pp. 106-107.

6 VielichKosky. B.; ZinCHenKo. V.; LURIA. A.: «Psicología de la percepción». Editorial Universidad de La Habana, 1982, en Bello. Z.: Psicología general. Editorial Félix Varela. La Habana, 2007 pp. 107-109.

7 VIELICHKOSKY. M, ZINCHENKO. V, LURIA. A.: «Psicología de la percepción». Editorial Universidad de La Habana, 1982 en Bello. Z.: Psicología general. Editorial Félix Varela. La Habana 2007 pp. 109-112. 
Igualmente, la constancia perceptiva está íntimamente ligada a la integridad. Ella se entiende como la independencia relativa de las características del objeto percibido respecto a su representación en la superficie receptora. Gracias a esta, los objetos se perciben como relativamente estables en cuanto a una forma, tamaño, color, jugando un papel muy importante en los procesos de adaptación.

Por último, el carácter objetal de la percepción puede expresarse además en la racionalidad. Este proceso no es más que la categorización del objeto percibido y la designación del mismo por medio de una palabra. En la racionalidad no solo se refleja el objeto de la realidad, sino su significación, que ha sido elaborada por la sociedad durante su desarrollo.

Otros autores 8 definen a la percepción como el resultado de un procesamiento de información que consta de estimulaciones a receptores en condiciones que se deben parcialmente a la propia actividad del sujeto.

BARTHEY ${ }^{9}$ por ejemplo, alega que la percepción es:

«Cualquier acto o proceso de conocimiento de objetos, hechos o verdades, ya sea mediante la experiencia sensorial o por el pensamiento; es una conciencia de los objetos, un conocimiento. La referencia que una sensación hace a un objeto externo. Un conocimiento inmediato o intuitivo, o juicio; un discernimiento análogo a la percepción sensorial con respecto a su inmediatez y al sentimiento de certidumbre que lo acompaña, frecuentemente implica una observación agradable o una discriminación sutil.»

Con estas definiciones el autor concibe a la percepción tanto como una forma de pensamiento como una conducta inmediata; un problema sensorial pues posee una naturaleza psíquica y es una copia de la realidad externa; y por último como un juicio, pues se da no solo a partir de datos sensoriales sino también como pura intuición ${ }^{10}$.

BRUNER Y COLBS. ${ }^{11}$ afirmaron que la percepción comprende fundamentalmente dos procesos. El primero consiste en la remodificación o selección del enorme caudal de datos que nos llegan del exterior, redu-

8 ARIAS. C.: Enfoques teóricos sobre la percepción que tienen las personas 2006 En https://dialnet.unirioja.es/descarga/articulo/4907017.pdf

9 BARTHEY. S.: «Principios de la percepción». Editorial Trillas. México D.F 1982, en ARIAS, C.: Enfoques teóricos sobre la percepción que tienen las personas 2006 p. 10 En https://dialnet.unirioja.es/descarga/articulo/4907017.pdf

10 ArIAS. C.: ob. cit. p. 10.

11 ÍDEM 
ciendo su complejidad y facilitando su almacenamiento y recuperación en la memoria, y el segundo, un intento de ir mas allá de la información obtenida, con el fin de predecir acontecimientos futuros y de ese modo, evitar o reducir la sorpresa.

Dichos procesos develan el carácter activo que tienen los sujetos en la formación de las percepciones. Ellos producen la información a la vez que crean las condiciones bajo las cuales se genera esta información.

NeISSER, representante de la Psicología Clásica, concibe a la percepción como un proceso activo-constructivo, en el que el perceptor, antes de procesar nuevas informaciones, y archivando los datos en su conciencia, es capaz de construir esquemas informativos anticipatorios que le permiten diferenciar los estímulos y aceptarlos o rechazarlos según se adecuen o no a las construcciones anticipatorias que realizó. Estas acciones el individuo es capaz de realizarlas en gran medida gracias al aprendizaje y las experiencias previas ${ }^{12}$.

Se considera además a la percepción como un proceso por fases ${ }^{13}$, en el que una imagen mental se forma a partir de las experiencias y necesidades de los sujetos, y pueden confluir en él la selección ${ }^{14}$, la organización ${ }^{15}$ y la interpretación ${ }^{16}$ de sensaciones de un modo jerárquico.

Hasta aquí podemos resumir que la percepción es un proceso activo, pues los sujetos producen la información a la vez que crean las condiciones bajo las cuales se genera esta información; histórico pues los seres humanos, en los propios procesos de interacción social, las han ido aprendiendo y adecuando a la realidad; con un carácter objetal, al ser una copia fiel del medio externo y reflejar como percepciones a los propios objetos de la realidad; selectiva, ya que el sujeto no puede percibir todo al mismo tiempo y por ello, selecciona su campo perceptual en función de lo que realmente desea percibir, mediatizando en este proceso de selección su propia subjetividad; y por último, un proceso temporal, al ocurrir en cortos plazos de tiempo, evolucionando a

12 : La percepción. Resumen. 2018, p. 1. En https://www.um.es/docencia/ pguardio/documentos/percpecion.pdf

13 Ídem.

14 Selección: se perciben parte de los estímulos que se reciben de acuerdo con las características personales de los individuos. Generalmente los mensajes se perciben de forma distorsionada, captando solo los aspectos agradables o los que responden a sus necesidades e intereses.

15 Organización: los estímulos seleccionados se organizan y clasifican en la mente de los individuos configurando un mensaje.

16 INTERPRETACIÓN: esta fase proporciona significación a los estímulos organizados. La interpretación depende de los factores internos de la persona, de su experiencia e interacción con el entorno. 
medida que aparecen nuevas experiencias o varían las motivaciones o necesidades de los sujetos.

\subsection{Percepción social}

Durante los años 50, la escuela de la Gestalt abordó el término de percepción social para profundizar en su estudio y convertirlo posteriormente en una de las grandes líneas que utilizaron en sus investigaciones. Los psicólogos discípulos de esta escuela comenzaron a plantear que las leyes que se habían enunciado para explicar la percepción de los objetos físicos, eran de igual forma aplicables a la percepción de las personas y del comportamiento social'17.

FRITZ HEIDER ${ }^{18}$ fue uno de los principales autores sobre las teorías de la percepción social. En sus trabajos, siempre tuvo como objetivo analizar la forma en que las personas perciben las relaciones interpersonales.

Según él, las personas tienden a observar su medio de forma organizada, como un todo estructurado y coherente, con una tendencia a percibir determinadas características como si fueran propiedades permanentes de los objetos. Estas percepciones van más allá de los datos conductuales, intentando encontrar relaciones que puedan ayudar a explicar los acontecimientos cambiantes. En sus argumentos sostiene que la forma en que percibimos el mundo social es el reflejo de una psicología ingenua, que nos orienta en nuestras relaciones con los demás, permitiéndonos predecir las acciones de otras personas e influir en las mismas.

Otro investigador del tema, Jerome Bruner planteó que «el término percepción social ha llegado a usarse ampliamente para describir la forma en que una persona percibe o infiere los rasgos e intenciones de otra, y hay un flujo constante de estudios experimentales sobre el modo en que los factores sociales provocan tipos de selectividad respecto de los que una persona percibe e infiere y respecto a su forma de interpretarla» ${ }^{19}$.

Este criterio coincide con el de ARIAS ${ }^{20}$ pues ella entendía que la percepción social se utilizó primeramente para indicar la influencia de los

17 Gómez. G. ob. cit., p. 20.

18 ÍDEM

19 Bruner s/f, citado por Torregrosa. J. y Crespo. E.: Estudios básicos de Psicología Social. Centro de Investigaciones Sociológicas, Barcelona 1984 p. 143 en Gómez. G ob. cit., p. 21

20 ARIAS. C. ob. cit., p.10. 
factores sociales y culturales en la percepción; la forma en que el medio social afectaba los procesos perceptuales. Luego el campo se extendió e incluyó los mecanismos de percepción de los otros, la formación de impresiones ${ }^{21}$, el reconocimiento de las emociones, la percepción que el individuo tiene de su medio físico y social, y más recientemente, el mecanismo de la atribución 22 .

Ambos autores reconocen la importancia del medio, de los factores sociales en la construcción de las percepciones de los individuos, sin anular otros elementos que pueden resultar vitales como el papel de los otros, las impresiones que de estos se construyen, así como las formas que buscamos para explicar las conductas de estos.

Para estudiar la percepción social es necesario tener en cuenta que el investigador constantemente está trabajando con experiencias privadas de las personas. Ella en sí misma constituye un constructo psicológico, un mecanismo hipotético que se emplea para explicar ciertas relaciones entre la entrada y la salida de los estímulos y la conducta manifiesta ${ }^{23}$. Este proceso está determinado en su totalidad por necesidades, valores sociales, motivaciones, metas, experiencias, familiaridad, aprendizajes y en general por las características permanentes y temporales de los individuos ${ }^{24}$.

Haciendo un breve recorrido por investigaciones realizadas en Cuba sobre el tema, emergen autores como Perera ${ }^{25}$; González-GuIJA ${ }^{26 ;}$ PALA-

21 En la FORMACIÓN DE IMPRESIONES, las personas se esfuerzan por formarse una impresión de otras personas; se tiende a completar la escasa información percibiendo a la persona como una unidad; las mismas cualidades podrían producir impresiones diferentes pues estas interactúan entre si dinámicamente y son capaces de producir una nueva cualidad; en ellas podemos encontrar una estructura, pues se organizan por cualidades centrales y cualidades periféricas (Asch, 1952 en Arias 2006, p. 12).

22 MeCANISMo o TeOría de la Atribución: Analiza la forma en la que explicamos la conducta de las personas. Por medio de esta se busca darle sentido al mundo; las acciones de las personas son atribuidas a causas internas y externas; se hace de manera bastante lógica y consistente y mediante ella las personas explican los acontecimientos cotidianos. (Myers. D.: Psicología Social. Percepción e interpretación de eventos. McGrw Hillcompanies, Inc, Bogotá, 2000 en ArIAs. C. 2006 ob. cit. p. 12).

23 Dember. W y Warm. J.: Psicología de la percepción. Alianza Editorial, Madrid 1990 p. 27 en ArIAS. C. ob. cit. p.10.

24 Morales. F; Moya. M.: Psicología Social. McGraw Hill. Madrid 1999 citado por Arias. C.: ob. cit. p. 20.

25 Perera. M.: Informe Final del proyecto de Investigación Percepción Social del Dirigente: Programa Nacional de Ciencia y Técnica. «El trabajo con los cuadros. (Trabajo inédito). Centro de Investigaciones Psicológicas y Sociológicas. La Habana, 2002

26 GonzÁlez-Guija.: La influencia de los medios en la percepción social de la delincuencia ejercida sobre la mujer. En soporte digital. s/f. 
$\mathrm{CIO}^{27}, \mathrm{GómeZ}^{28}$. Todos ellos estudian a la percepción social desde diferentes puntos de vista, haciendo importantes aportes en cada caso al fenómeno perceptual que se investiga.

La Dra. C. Maricela Perera ${ }^{29}$ por ejemplo, declara que:

«... una percepción es un enunciado, un juicio que denota la presencia de criterios, que configurados con mayor o menor información y elaboración, denota los sentidos personales, los cuales se expresan a través de los discursos y acciones de los sujetos individuales o colectivos sobre cualquier hecho, evento u objeto social, relacionado directa o indirectamente con los mismos. Se constituyen a través de las acciones, relaciones y cogniciones de los hombres, son ínter e intrasubjetivas al surgir de la influencia de la sociedad sobre una individualidad única e irrepetible, a partir de una experiencia vital concreta en un contexto sociohistórico particular.

A partir de estos presupuestos define a la percepción social entonces como:

«(...) una dimensión de la subjetividad configurada en el contexto interaccional de los individuos y sus grupos. En ella se integran los significados, que de una forma más o menos consciente, el sujeto confiere a los objetos de su percepción, así como la relación que a partir de dichos significados, establece entre medios y fines de sus acciones, respecto al objeto de su percepción. ${ }^{30}$

Fruto de esta relación social pueden surgir percepciones tanto coincidentes como divergentes. Las primeras muestran esencias comunes, a pesar de la diversidad de expresiones o términos que se empleen para explicitarlas, mientras que las segundas no tienen un núcleo común con otras sino que muestran nuevos contenidos perceptuales sobre el fenómeno 31 .

Por tanto, podemos concluir que a partir de la percepción social, los individuos no somos meros reproductores, sino creadores de nuestros propios sistemas cognoscitivos. En la interacción con los otros o a partir de nuestras motivaciones, necesidades, metas e intereses expre-

27 PALACIO, Y.: Identidad nacional y cine cubano contemporáneo: una visión desde la subjetividad del espectador. Tesis en opción al grado de Máster en Psicología Social y Comunitaria. Universidad de La Habana, 2005.

28 Gómez. G. ob. cit.

29 Perera. M. ob. cit. en Gómez. G. ob. cit., pp. 22-23.

30 ÍDEM.

31 Gómez. G ob. cit. p. 95. 
samos lo que sentimos, nos formamos impresiones sobre los demás, encontramos respuestas a sus conductas, le damos sentido al mundo y nos explicamos los hechos que acontecen a diario en nuestra vida cotidiana.

\subsection{Percepción social y cooperativas}

A nivel mundial, desde hace algún tiempo se vienen desarrollando investigaciones que tratan este tema desde diversos puntos de vista. GARGALLO Y FREUNDLICH ${ }^{32}$ —por ejemplo- analizaron las percepciones de los socios y no socios cooperativistas sobre la satisfacción laboral. Aplicando una encuesta a 250 trabajadores de una cooperativa de gran tamaño, concluyeron que existen diferencias en las percepciones de los trabajadores socios y no socios sobre los determinantes de la satisfacción laboral. Con su estudio, permitieron establecer recomendaciones para favorecer actuaciones encaminadas a su mejora.

Igualmente BELATEGI, GAGO Y EGAÑA ${ }^{33}$ estudiaron la comunicación interna en las cooperativas a partir de la percepción de las personas trabajadoras sobre la escucha y la información. Su propósito fue conocer, en una cooperativa del Grupo Mondragón, por un lado, las percepciones de las personas trabajadoras sobre la información transmitida por la cooperativa; por otro lado, sus percepciones sobre cómo realizan la escucha los directivos y mandos intermedios cuando les trasladan sus preocupaciones y expectativas.

En Cuba, a pesar de la poca representatividad de los estudios relacionados con el tema, destacan algunos que han contribuido a profundizar en la percepción social y las cooperativas no agropecuarias. Tal es el caso de IGLESIA y BARCENAS, los cuales desarrollaron una investigación sobre la percepción social de las cooperativas en Cuba, enfatizando en la Cooperativa de Servicios Contables ACERCO. Este acercamiento les permitió caracterizar la percepción social de asociados y clientes de la cooperativa, concluyendo que existen diferencias entre

32 Gargallo. A, Freundich. F.: «Percepciones de los socios y no socios cooperativistas sobre la satisfacción laboral». REVESCO. Revista de Estudios Cooperativos No. 103. (2010) pp. 33-58. En https://revistas.ucm.es/index.php/REVE/article/view/ REVE1010440033A/18585

33 Belategi. O., Gago. M., Egaña. T.: «La comunicación interna en las cooperativas: la percepción de las personas trabajadoras sobre la escucha y la información». REVESCO. Revista de Estudios Cooperativos, Vol. 130 (2019). pp. 9-32. En http://dx.doi. org/10.5209/REVE.62812 
las percepciones de los socios y los clientes, aunque no muy significativas en cuanto al conocimiento que poseen de las cooperativas como nueva forma de gestión no económica. Concluyeron además que se considera a la cooperativa como una organización y asociación que en el contexto actual cubano constituye una solución, tanto a nivel individual como social. No obstante, se evidencia incertidumbre, desconocimiento, falta de apoyo y la inexistencia de condiciones reales para su funcionamiento, por lo que genera malestar a nivel subjetivo. Además, se percibe la calidad de los servicios de la cooperativa y el apoyo que constituyen para el sector estatal, aunque señalan que deben regularse y controlarse según su objeto social en nuestro contexto. ${ }^{34}$

Por otro lado EsTUPINAN ${ }^{35}$ centró su atención en determinar la percepción social de la cooperación de los cooperativistas no agropecuarios de Santa Clara. Aquí ella logra caracterizar las percepciones sociales que de la variable cooperación han configurado los cooperativistas no agropecuarios de la ciudad de Santa Clara. Los resultados que obtuvo la investigadora demostraban que las mismas se configuraban en torno a dos núcleos de sentidos: las percepciones de intracooperación y las percepciones de intercooperación. ${ }^{36}$

Es constatable, luego de este breve recorrido por las principales investigaciones en torno a la percepción social y las cooperativas, que el tema aún carece de sistematización por parte de las ciencias sociales. Los estudios realizados hasta la fecha son escasos, tanto en contextos nacionales como foráneos. Falta lograr una representatividad de las muestras, pues si bien es cierto que en ocasiones pueden llegar a ser extensas, se corre el riesgo de excluir a socios o cooperativas que pudieran hacer valiosos aportes a la investigación. Así mismo creemos necesaria la intervención de otras variables que develen, con más precisión y representatividad las percepciones de socias y socios. Este diagnóstico, precisamente pretende dar un primer paso en ese sentido. El mismo procura ser el inicio de una serie de investigaciones que profundicen en la percepción social de los asociados respecto a cooperativas, a fin de contribuir a crear espacios de satisfacción y realización.

34 IGLESIAS. E.; BARCENAS. J.: «Un estudio sobre la percepción social de las cooperativas en Cuba». Cooperativa de servicios contables ACERCO. En Evento Internacional de Psicología «Personas de Miradas Diversas». Universidad de Pinar del Río 2017

35 Estupiñan. B.: Percepción social de la cooperación de los cooperativistas no agropecuarios de Santa Clara. Tesis de Diploma. Facultad de Ciencias Sociales. Universidad Central «Marta Abreu» de Las Villas, Cuba 2017 En http://dspace.uclv.edu.cu/bitstream/ handle/123456789/8413/OKp5.pdf?sequence=1\&isAllowed=y

36 Ídem. p. 60. 


\section{Cooperativas no Agropecuarias en Cuba. Especial referencia a la provincia de Pinar del Río}

En Cuba, hasta hace unos pocos años, el sector cooperativo nacional se desarrolló solamente en la esfera agropecuaria de la economía. No fue hasta abril de 2011, con la celebración del VI Congreso del Partido Comunista de Cuba, que se aprueban los Lineamientos de la Política Económica y Social del Partido y la Revolución, destinados a sentar las pautas necesarias para conducir el proceso de actualización del modelo económico socialista en el país. ${ }^{37}$

Según el Lineamiento 2, el modelo reconocerá y promoverá las cooperativas en diferentes sectores de la economía, en correspondencia con los cambios que se propone realizar. El lineamiento 25 plantea que:

«Se crearán las cooperativas de primer grado como una forma socialista de propiedad colectiva, en diferentes sectores, las que constituyen una organización económica con personalidad jurídica y patrimonio propio, integradas por personas que se asocian aportando bienes o trabajo, con la finalidad de producir y prestar servicios útiles a la sociedad y asumen todos sus gastos con sus ingresos. ${ }^{38}$

Se subraya además que se crearán cooperativas de segundo grado, cuyos socios son cooperativas de primer grado, las que tendrán personalidad jurídica y patrimonio propio y se forman con el objetivo de organizar actividades complementarias afines o que agreguen valor a los productos y servicios.

Sobre esta base, se crean condiciones mínimas desde el punto de vista jurídico-institucional para fomentar e implementar, con carácter experimental, cooperativas más allá del sector agropecuario de la economía 39 .

Desde principios de 2013 y hasta el momento se ha autorizado la creación y funcionamiento de 398 cooperativas en Cuba. El 89 \% se

37 Partido Comunista de Cuba: Lineamientos de la Política Económica y Social del Partido y la Revolución, aprobados el 18 de abril de 2012. Visto en http: //www. prensalatina.cu/Dossiers/LineamientosVICongresoPCC.pdf

38 Partido Comunista de Cuba 2011 en Ponce. F., Perugorría. D., Salgado. R.: Las cooperativas no agropecuarias en la actividad de los servicios técnico personales y de uso doméstico en la provincia de Pinar del Río: Desarrollo, Limitaciones y propuestas para perfeccionar su gestión s/f. Consultado en https://dialnet.unirioja.es/descarga/ articulo/5294661.pdf

39 Gaceta Oficial No. 53, Extraordinaria, de 11 de diciembre de 2012. 
concentra en cinco sectores: Gastronomía 151 (38\%), Comercio 81 (20\%), Construcción 59 (15\%), Industria 34 (9\%), Servicios personales y técnicos 29 (7\%), Ornitología 17, Transporte 12, Alimentaria 3, Energía 7, Servicios contables $5^{40}$.

Por otro lado, es necesario mencionar que la provincia de Pinar del Río cuenta con una tradición histórica, al ser pionera en la implementación de las cooperativas, agrupadas todas en el sector de la agricultura, desde los propios inicios de triunfo de la revolución ${ }^{41}$ y luego una de las primeras en el país en materializar la expansión de las cooperativas hacia otros sectores de la economía diferentes al agropecuario.

Dicho territorio cuenta con un Centro de Estudios de Desarrollo Cooperativo y Comunitario (CEDECOM) devenido en Centro de Estudios de Dirección, Desarrollo Local, Turismo y Cooperativismo (CEGESTA), en la Universidad de Pinar del Río (UPR), que ha funcionado como un referente a nivel nacional para el proceso de expansión de las cooperativas a otras esferas de la economía diferentes a la agropecuaria. ${ }^{42}$

Igualmente en la UPR se han creado otros espacios que se proponen perfeccionar las dinámicas de las cooperativas no agropecuarias (CnoA) e impulsar su desarrollo. Tal es el caso del Departamento de Derecho de la Facultad de Ciencias Sociales y Humanidades, el cual, hace algunos años, viene desplegando una serie de acciones investigativas con el fin de potenciar el trabajo en y hacia estas empresas. En este sentido, es imperante mencionar sendos proyectos de investigación que han alcanzado relevantes resultados desde su implementación. El primero, ejecutado desde el año 2013 hasta el 2016, se centró en fundamentar una propuesta de "Bases Teóricas para la efectiva expansión jurídica de las cooperativas hacia otras esferas de la economía nacional además de la agropecuaria», logrando importantes resultados: una Tesis de Doctorado en Ciencias Jurídicas; 20 publicaciones en libros o revistas científicas; 33 participaciones en eventos científicos, la mayoría de ellos internacionales; 1 Grupo Científico Estudiantil; 4 Tesis de Grado en Licenciatura en Derecho; 2 ediciones de un evento internacional propio con proyecciones futuras; cursos de posgrado; varios premios a sus resultados además de vínculos con instituciones tanto

40 Fuente: COMISIÓN DE IMPLEMENTACIÓN. Tomado de https://www.cubadebate.cu/ noticias/2019/08/30/nuevas-normas-juridicas-para-las-cooperativas-no-agropecuariasen-cuba/\#.XoNx2SXB-Ec

41 RodríGuez. O: La Constitucionalización de la cooperativa. Una propuesta para su redimensionamiento en Cuba, Vincere Editora, Brasília-DF, 2017, p. 112.

42 Ídem p. 22. 
nacionales como internacionales, con conocido prestigio en el campo, dispuestas a continuar colaborando en el estudio de las CnoA cubanas.

Entretanto, el segundo proyecto ya cuenta con un año de implementación, y este, a partir de un convenio con la Empresa de Servicios Legales de Pinar del Río (EPSEL), se ha propuesto crear una «Metodología para perfeccionar el proceso de Asesoría Jurídica de las Cooperativas No Agropecuarias en la provincia de Pinar del Río, desde su gestación hasta su disolución».

Pese a su corto período de ejecución, ya exhibe importantes resultados: 9 publicaciones científicas; 4 ponencias en eventos científicos —nacionales e internacionales -; 6 reuniones metodológicas que han aglutinado tanto a los profesores-investigadores como a los asesores jurídicos de las CnoA de la provincia; 2 cursos de posgrado; 1 Premio Nacional; 1 estancia de investigación; 3 capacitaciones y 1 tesis de maestría en curso.

Su ejecución ha posibilitado además profundizar en temas como la responsabilidad social cooperativa, el control democrático de los asociados, la educación, capacitación e información cooperativa, la intercooperación entre cooperativas, la participación económica de los socios, y la perspectiva de género que se tiene en las cooperativas no agropecuarias en Pinar del Río, todo ello con el fin de diagnosticar limitaciones en la metodología empleada por los consultores jurídicos del territorio al brindar asesoría a las CnoA.

Estas acciones se han implementado en las 12 CnoA (Cooperativas no Agropecuarias) que actualmente existen en la provincia: Café Pinar (ofrece servicios gastronómicos entre otros), Combinado Industrial de Pinar del Río (produce y comercializa muebles y artículos de madera), Electrónica Hermanos Cruz (brinda servicios de reparación, mantenimiento y diagnóstico de equipos eléctricos y electrodomésticos), TaIler de Reparación de Calzado «Estrella Roja» (repara calzados, carteras, maletines, además del lustrado de calzado), Clavel (brinda servicios gastronómicos), Ornitología Pinar del Río (brinda servicios de acopio y comercio de aves), Restaurant Cielo Azul (brinda servicios de gastronomía), Casa Colonial (brinda servicios de restaurant), Atelier «La Moda» (confecciones y arreglos de atelier y sastrería), Recuperación de desechos de Viñales (recuperación y reciclaje de desechos), Carpintería Sandino (servicios de carpintería).

Los datos muestran una composición heterogénea por sectores, encontrando diversidad en los servicios que ofertan, así como en el objeto social de cada una. Estructuralmente pueden considerarse cooperativas pequeñas, pues la mayoría no excede la cifra de 20 socios, y en todas encontramos representación de los cargos de presidente, sustituto del 
presidente, secretario, administrador y comisión de control y fiscalización. Por último consideramos importante agregar que existe una mayor representación de hombres que de mujeres, tanto en la estructura interna de las CnoA pinareñas como en la asunción de cargos de dirección para garantizar el funcionamiento de las mismas.

Para aprovechar las potencialidades del territorio, hemos decidido intencionar nuestra mirada de investigadores hacia esta provincia, con el fin de profundizar en el estudio de las cooperativas no agropecuarias que allí existen. Desarrollar esta investigación en Pinar del Río nos posibilitará la retroalimentación con las investigaciones realizadas hasta el momento, así como contar con el apoyo de las instituciones y grupos que sistemáticamente han estudiado a las CnoA.

\section{Percepción Social de los asociados a cooperativas no agropecuarias cubanas. El caso del Atelier "La Moda»}

La escasa sistematización científica del tema no ha imposibilitado que se conozcan opiniones de los socios con respecto a las cooperativas que los agrupan. Diversos trabajos así lo demuestran ${ }^{43}$. En ellos, las aproximaciones con los socios muestran algunas de las percepciones que han elaborado sobre las CnoA. Consideran que a partir de su surgimiento:

- Aumentan sus ingresos, triplicándose como promedio.

- Incrementa la motivación, pues se reflejan cambios en la actitud ante el trabajo, mejorando la disciplina laboral, disminuyendo el ausentismo y los errores técnicos, se cuidan mejor los medios de trabajo, dándoles mantenimiento cuando lo necesitan.

- Mejora el ambiente laboral pues han experimentado progresos tanto en las condiciones materiales de trabajo como en cuestiones más subjetivas pero importantes como las relaciones laborales.

- Existencia de trabas para relacionarse con entidades estatales.

- Carencia de tiendas mayoristas para la compra.

43 Extremera. D.: "Cooperativas de la construcción en Cuba: cinco años después 2018». En http://www.cubadebate.cu/?s=Cooperativas; SIFONTE. Y.: Cooperativas-Empresas: Los eslabones perdidos de una cadena. 2019. En http://www.cubadebate.cu/ especiales/2019/04/04/cooperativas-empresas-los-eslabones-perdidos-de-una-cadena/\#. XoOMWSXB-EC; PINEEIRO. C.: «Nuevas cooperativas cubanas: logros y dificultades». En Miradas a la Economía Cubana: Análisis del Sector No Estatal, Ed. Caminos, La Habana, 2015, pp. 51-61. 
- Existencia aún de mecanismos burocráticos que subsisten entre una empresa estatal y una cooperativa.

- Limitada participación de los socios en las decisiones y asambleas generales.

- Complejo y demorado proceso de aprobación de las cooperativas.

- Limitaciones en el acceso al mercado o las irregularidades propias de este proceso.

- Discriminación a la cooperativa como ente económico.

- Poco acceso a empresas importadoras.

- Existencia de prejuicios con relación a las cooperativas y sus socios (asociados a la malversación, el robo).

- Falta de capacitación y orientación hacia temas cooperativos.

— Falta de involucramiento de los gobiernos locales.

Los criterios expuestos son un reflejo de la realidad que vivencian cotidianamente. Los asociados en estos trabajos, muestran opiniones tanto positivas como negativas en torno a las cooperativas no agropecuarias. Sus juicios desde un plano individual, se van configurando a partir de las experiencias, los aprendizajes y la familiaridad que poseen con su objeto perceptual.

En el caso que nos ocupa vemos cómo los socios de las 12 CnoA del territorio pinareño ${ }^{44}$ interpretan su realidad, y la traducen en percepciones que en la mayoría de las ocasiones son coincidentes con las del resto del país, por ejemplo:

- Desconocían lo que era una cooperativa y sus funciones al asociarse.

- Se realizaron pocas acciones de capacitación a los socios al momento de asociarse.

- Las mayores motivaciones para asociarse en cooperativas se derivaban de la prosperidad económica y el mantenimiento de su trabajo.

- Perciben una exigua participación como socios en la construcción de los Estatutos de las CnoA.

- Describen de igualitaria y colectiva la toma de decisiones de la cooperativa.

- Aún mantienen dinámicas de la empresa estatal en las relaciones socios-dirigentes, faltando estrategias bien diseñadas que garanticen el desarrollo de las CnoA y la superación de estos modos de funcionamiento.

44 Vid. epígrafe II. 
- Catalogan de insuficientes las gestiones de la cooperativa para capacitar y educar a sus socios.

- Refieren que es escasa la cooperación y colaboración entre cooperativas, tanto a nivel territorial como nacional.

- Reclaman un aumento en la contribución de las cooperativas al desarrollo de su comunidad.

- No existe una proyección hacia mejorar la imagen comercial de las cooperativas.

- El cambio a cooperativa ha posibilitado explotar todas las potencialidades como empresa y consolidar un pensamiento emprendedor.

- Identifican a CnoA que desde su constitución, se han proyectado con mayor agresividad hacia el entorno empresarial y del mercado.

- Perciben igualmente un aumento del trabajo y vínculos con la universidad. Los socios, en su mayoría, lo definen como facilitador y formativo.

- Opinan que los controles y controladores externos no se adaptan al funcionamiento de una cooperativa, sino que los practican a partir de las experiencias de la empresa estatal y en ocasiones se viola la autonomía de la cooperativa.

- Identifican aún en algunos decisores una falta de conocimiento y pensamiento cooperativo, que a su consideración, contribuye a que en ocasiones sean hostiles con esta forma de gestión.

Desde luego, aquí solo se describen algunos de los contenidos perceptuales identificados en un primer diagnóstico. El necesario acercamiento al tema en la provincia y el país nos convocó a darle una mirada más profunda, esta vez centrándonos en solo una cooperativa no agropecuaria, en la que partiendo de sus singularidades, identificaríamos y caracterizaríamos la percepción social de los asociados a partir de sus criterios, necesidades y experiencias.

Teniendo en cuenta entonces la trayectoria destacada de la CnoA Atelier «La Moda» en la implementación de este modelo de gestión no estatal en Pinar del Río, decidimos utilizarla como muestra en la exploración de la percepción social de sus trabajadoras. Antes de profundizar en los resultados obtenidos, nos aproximaremos brevemente a datos generales de esta CnoA.

La cooperativa Atelier «La Moda» está enclavada en el municipio San Luis. Fue constituida como una cooperativa de primer grado, mediante escritura pública ante notario el 13 de septiembre de 2013. Desde sus inicios, el objeto social que le fue autorizado consistió en 
«prestar servicios de confecciones y arreglos de atelier y sastrería, alquiler de prendas de vestir y otros que complementen este servicio, confecciones de ajuares del hogar, confecciones de muñequerías, carteras y otros objetos utilitarios, montaje de cortinas, mantelería, adornos florales, decoraciones y otros vinculados a la actividad de los atelieres y venta de ropa reciclada asociada a los servicios de atelier». Se estableció, además, que las operaciones que se realizaran debían hacerse en CUP, que se diera en arrendamiento el inmueble que ocupaba el Atelier "La Moda», que se le vendieran los medios, utensilios y herramientas disponibles para el desarrollo de la actividad y que los insumos —entiéndanse tejidos, accesorios y útiles de servicios - se le proveerían «a través del canal de distribución actual e la Empresa de productos Universales» ${ }^{45}$.

Según los Estatutos de la Cooperativa en Formación esta organización se creó por ocho (8) socias, todas mujeres, para una duración de 10 años, con un capital de trabajo inicial de 1350 CUP, siendo el aporte de cada socia de 168,73 CUP. Los órganos de dirección adoptados fueron dos: la Asamblea General y la Comisión de Control y Fiscalización, integrada por tres (3) socias.

A partir de que la entidad adoptara la nueva forma de gestión cooperativa, mantuvo su interés por apoyar los programas de desarrollo territorial. Esto contribuyó a aumentar el grado de satisfacción de la demanda social unido a un elevado nivel de aprovechamiento de sus capacidades y recursos.

Otro elemento de importancia es su dualidad como organización económica con elevada finalidad social, la cual debe lograr ser eficiente y competitiva en ambos aspectos; solo así se conseguirá conjugar la esencia humanista del cooperativismo con el éxito empresarial.

Como la cooperativa es por su propia naturaleza una institución social, dentro de sus planes estratégicos se incorporaron políticas concretas de ámbito interno y externo que tienen por objeto desarrollar proyectos comunitarios en colaboración con otras entidades del territorio.

La CnoA, además ha servido de muestra en el desarrollo de varias investigaciones, sobre todo las relacionadas con el enfoque de género y las cooperativas en Cuba. Destacan en esta línea las realizadas por HeRNÁNDEZ en diversos años ${ }^{46}$.

45 Consejo de la Administración Provincial, 2013.

46 HeRnández. O.: «La relación género y cooperativa en el ordenamiento jurídico cubano. Una aproximación a su realidad y sus perspectivas ante el proceso de perfeccionamiento del modelo económico en el país». Boletín Anual de la Asociación Internacional de Derecho Cooperativo, Deusto, No. 46, 2012 pp. 297-320. En http://baidc.revistas. deusto.es/article/view/140 
Particularizando aún más en las características de esta CnoA, y desde el acercamiento realizado durante la investigación, conocimos que las 8 socias con que cuenta se ubican en un nivel de escolaridad que oscila de medio, técnico medio y medio superior, no existiendo socias graduadas de un nivel superior (universitario). Ello puede estar relacionado con la actividad que desempeñan, pues históricamente los arreglos de atelier y sastrería no han exigido de sus practicantes un alto nivel escolar. Igualmente, en nuestro país este oficio se potencia a partir del egreso de la secundaria básica, por lo que su aprendizaje se consolida desde un técnico medio o escuela de oficios.

Las edades más representativas de la cooperativa oscilan entre 51 y 60 años, denotando este dato la presencia de mujeres con una alta experiencia en el oficio, lo que le otorga a esta forma asociativa un nivel de experticia que les permite alcanzar buenos resultados en la producción y los servicios que brindan.

Al explorar las percepciones que poseen las socias en torno al modo de creación de la cooperativa, nos percatamos que de comúnmente todas coinciden al afirmar que más que una iniciativa propia, su surgimiento fue condicionado a la decisión de una organización superior, anulando la iniciativa libre y espontánea de las trabajadoras para asociarse. A decir de Rodríguez ${ }^{47}$ «... esta falta de voluntariedad real (vs. formal) conspira contra la identidad universalmente reconocida a las cooperativas, afectando concretamente el principio de "asociación voluntaria», cuyo irrespeto puede acarrear consecuencias irreversibles para la estabilidad del sector y para su credibilidad social, en tanto resulta premisa esencial de su efectiva consolidación...»

Pese a lo anterior, sus criterios respecto al grado de satisfacción con la cooperativa oscilan en su mayoría desde satisfechas hasta muy satis-

Hernández. O.: "La relación género y cooperativa en Cuba. Perspectivas dentro del marco jurídico experimental de la cooperativa». Revista de Estudios Cooperativos, No. 2, 2013 pp. 133-156. En https://dialnet.unirioja.es/servlet/articulo?codigo=4191082

HeRNÁNDEZ. O.: "Las cooperativas no agropecuarias en Pinar del Río. Una aproximación al enfoque de género en su funcionamiento». Revista Deusto Estudios Cooperativos No. 6, 2015 pp. 159-179. En https://dialnet.unirioja.es/servlet/articulo?codigo=5205846

Hernández. O., Valle. D.: «La Cooperativa No Agropecuaria Atelier "La Moda": una aproximación a la experiencia de una cooperativa de mujeres». En Congreso Nacional de Estudios del Trabajo: El trabajo en conflicto: Dinámicas y expresiones en el contexto actual, Buenos Aires, 2017. En https://www.aset.org.ar/2017/ponencias/11_Hernandez_ Aguilar.pdf

HeRnÁndez. O.: «Consideraciones en torno al enfoque de género en las Cooperativas no Agropecuarias en Pinar del Río». Revista Deusto Estudios Cooperativos No. 12, 2019 pp. 141-166. En https://dialnet.unirioja.es/servlet/articulo?codigo=6887106

47 Rodríguez. O. ob. cit., p. 100. 
fechas. Los significados conferidos a la CnoA como objeto de percepción develan la existencia de una opinión positiva y a la vez son muestras del bienestar y la complacencia de las socias con su espacio laboral. Igualmente, estos significados aumentan su sentido de pertenencia y la cooperación en y hacia el trabajo.

Entretanto, las percepciones que giran alrededor de los estilos de liderazgo que practica la cooperativa podemos definirlas como coincidentes en su totalidad. El carácter objetal de estas percepciones se manifiesta a través de la racionalidad, puesto que categorizan al estilo de liderazgo como democrático e incluso marcan un antes y un después en su implementación al afirmar que constituirse como cooperativa condujo a un cambio obligatorio en la formas de dirección. Lo anterior se confirma a partir de expresiones como «... porque este negocio es de nosotros...» y porque "... se debe pensar como empresarios...», las cuales denotan sentidos personales hacia la CnoA, enfatizando en la colectividad, en lo grupal y común característico en las empresas del tercer sector.

De positivos también pudieran caracterizarse los criterios de las socias con respecto a los ingresos y salarios que proporciona la cooperativa. Desde su experiencia, la constitución como empresa ha favorecido el aumento - considerable - de su economía. Este factor, según RodRíGUEZ, «... se asocia directamente a que la sumatoria de anticipos y utilidades (como uno de los modos de funcionamiento en las cooperativas, y ha incrementado considerablemente sus beneficios económicos con respecto a los salarios que anteriormente recibían ${ }^{48}$ 》.

Es real además que al aumentar los beneficios económicos, aumenta la cantidad de trabajo de las socias, y la cooperativa aún no cuenta con un sistema de estrategias que permita enfrentar grandes demandas de trabajo sin sobrecargar la jornada laboral. En su funcionamiento aún no se apoyan en la contratación de personal externo para cumplir con los planes establecidos, sino que suman a las socias este trabajo adicional. De este modo, perciben que debido a las propias exigencias de una empresa cooperativa, el impacto del trabajo ha sido «peor» en su doble jornada laboral ${ }^{49}$.

48 ÍDEM. p. 115.

49 Doble JORNADA LABORAL: Trabajo doméstico al que se exponen en mayor medida las mujeres al concluir una jornada laboral, el cual demanda altas exigencias que lo pueden tornar, igual o más agotador que el trabajo profesional. Esta sobrecarga de tareas hace que la doble jornada laboral de la mujer incida en la posterior realización de su jornada laboral, lo que pudiera provocar en estas, problemas de salud psíquica o física. (VAlle. D.: Efectos de las exigencias laborales de una jornada de trabajo en la salud de profesores de la Facultad de Ciencias Sociales y Humanísticas de la Universidad de Pinar del Río. Tesis de Licenciatura. Facultad de Psicología, Universidad de La Habana 2014 p.35). 
Igualmente observan la inestabilidad del mercado para la adquisición de materias primas y servicios, factor que incide negativamente en los niveles de rendimiento que puedan tener en el trabajo que desarroIlan. Es una realidad cotidiana para las CnoA del país la inexistencia de un mercado mayorista donde comprar, según sus necesidades, los insumos de trabajo. Como se ha señalado, la «inexistencia de un sistema de abastecimiento en correspondencia con las demandas de las cooperativas que les permita obtener, a precios mayoristas (...) los indispensables insumos y las materias primas que necesitan, acarrea intermitencias, baja calidad o incremento en los precios del servicio ofrecidos, junto a la afectación de la imagen pública de estas nuevas formas de gestión» ${ }^{50}$.

Luego de este análisis podemos concluir que las socias de esta CnoA han configurado sus percepciones a partir de la creación y generación de la información que manejan, teniendo en cuenta sus necesidades, motivaciones y metas. Ello demuestra el carácter activo que han tenido en la construcción de la realidad que vivencian con respecto a su trabajo.

Sobre la base de los conocimientos, aprendizajes y experiencias adquiridos en este espacio laboral, los socios de todas las cooperativas no agropecuarias del país, han configurado juicios que llenan de significado y sentido personal a su objeto de representación. Estos criterios son positivos y negativos, en tanto algunos denotan un sentido de pertenencia y satisfacción con la cooperativa, otros resaltan elementos en los que es necesario trabajar aún más para que no afecten su calidad y rendimiento. El desarrollo de la investigación nos ha permitido sistematizarlos y organizarlos desde las propias percepciones de los socios. Para ellos, las CnoA han contribuido a:

- Aumentar la motivación hacia el trabajo.

- Hacer crecer la economía y los ingresos de los socios.

- Favorecer los altos grados de satisfacción y sentido de pertenencia de sus trabajadores.

- Fomentar y practicar la cooperación en el trabajo.

- Mejorar el ambiente laboral de los asociados.

- Lograr un trato igualitario y colectivo en la toma de decisiones de la cooperativa.

- Aprovechar más las potencialidades de una empresa.

- Consolidar el pensamiento emprendedor en los socios.

50 Rodríguez, O. y Rivera, J.: «Historia y realidad jurídica de la cooperativa en Cuba. Aproximación desde la experiencia práctica a las noveles cooperativas no agropecuarias en Pinar del Río». Boletín de la Asociación Internacional de Derecho Cooperativo, 49 (2015), p. 215. En http://baidc.revistas.deusto.es/article/download/757/916 
— Fortalecer los vínculos de trabajo con las universidades y Centros de Estudios del país.

Igualmente, desde un rol activo y crítico reconocen que:

- Su surgimiento estuvo condicionado a organizaciones superiores, anulando la libertad y voluntariedad de los socios para agruparse.

- Aún existen trabas en la relación empresa estatal-cooperativa.

- Carecen de tiendas mayoristas para realizar sus compras.

- Existen aún prejuicios con respecto a las CnoA.

- Escasa capacitación y educación a los socios.

- Poca colaboración y cooperación entre cooperativas.

- Poca autonomía cooperativa.

- Débil apoyo de alguno decisores públicos debido a su escaso conocimiento cooperativo.

- Aumento de la cantidad de trabajo y por ende, de las jornadas laborales para los socios.

Estos criterios nos indican la existencia de percepciones coincidentes en todos los socios, pues a pesar de que usan diferentes expresiones al caracterizar a sus cooperativas, dichas expresiones son comúnmente compartidas, y llegan a agrupar contenidos perceptuales similares.

\section{Conclusiones}

1. La percepción es un proceso activo, histórico, selectivo, temporal, con un carácter objetal. En la interacción con los otros o a partir de nuestras motivaciones, necesidades, o intereses, creamos nuestras propias percepciones, las que permiten que expresemos lo que sentimos, nos formemos impresiones sobre los demás, encontremos respuestas a sus conductas, le demos sentido al mundo y nos expliquemos los hechos que acontecen a diario en nuestra vida cotidiana. Tanto a nivel nacional como internacional, las investigaciones sobre percepción social y cooperativas son escasas y el ellas falta lograr sistematizar estudios de varias cooperativas así como intervenir con otras variables que develen, con más precisión y representatividad, las percepciones de socias y socios.

En Cuba, hasta hace unos pocos años, el sector cooperativo nacional se desarrolló solamente en la esfera agropecuaria de la economía. No fue hasta abril de 2011, con la celebración del VI Congreso del Partido Comunista de Cuba, que se aprueban los Lineamientos de la Política Económica y Social del Partido y la Revolución. 
2. En Cuba, a partir de 2013, surgen de forma experimental un nuevo grupo de cooperativas. Pinar del Río cuenta con una tradición histórica, al ser pionera en la implementación de las cooperativas agropecuarias desde los propios inicios de triunfo de la revolución y luego, una de las primeras en el país en materializar la expansión de las cooperativas hacia otros sectores de la economía diferentes al agropecuario. Esto, junto a la existencia en el territorio del Centro de Estudios de Dirección, Desarrollo Local, Turismo y Cooperativismo (CE-GESTA), y el Departamento de Derecho de la Facultad de Ciencias Sociales y Humanidades en la Universidad de Pinar del Río, ambos con amplia trayectoria en el estudio de estas empresas, permitió la investigación del tema específicamente en la provincia, profundizando en el estudio de las cooperativas no agropecuarias que allí existen.

3. A pesar de la escasez de investigaciones sobre el tema, se logran identificar algunas de las percepciones que tienen los socios sobre sus cooperativas. Estas constituyen un reflejo de la realidad que vivencian cotidianamente. Encontramos opiniones positivas y negativas en tanto algunos denotan un sentido de pertenencia y satisfacción con la cooperativa y otros resaltan elementos en los que es necesario trabajar con más sistematicidad para que no afecten su calidad y rendimiento. Los criterios de los asociados se van configurando a partir de las experiencias, los aprendizajes y la familiaridad que poseen con su objeto perceptual. A pesar de que usan diferentes expresiones para caracterizar a sus cooperativas, estas son comúnmente compartidas, y llegan a agrupar contenidos perceptuales similares, por lo que pueden definirse como percepciones coincidentes. En su estructura se reflejan necesidades, motivaciones y metas que dan cuenta del carácter activo que han tenido en la construcción de la realidad que vivencian con respecto a su trabajo.

\section{Bibliografía}

: La percepción. Resumen, 2018. En https://www.um.es/docencia/ pguardio/documentos/percpecion.pdf

ArIAS, C.: Enfoques teóricos sobre la percepción que tienen las personas 2006.

En https://dialnet.unirioja.es/descarga/articulo/4907017.pdf

Belategl, O., Gago, M., Egaña, T.: «La comunicación interna en las cooperativas: la percepción de las personas trabajadoras sobre la escucha y la información». REVESCO. Revista de Estudios Cooperativos Vol. 130 (2019). En http://dx.doi.org/10.5209/REVE.62812

Bello, Z., Casales, J.: Psicología social. Editorial Félix Varela. La Habana. 2005. 
Bello, Z.: Psicología general. Editorial Félix Varela. La Habana 2007.

Calviño, M. (coordinador): Descubriendo la Psicología. Editorial Academia. La Habana. Cuba. 2017.

Consejo de la Administración Provincial: Acuerdo N. ${ }^{\circ}$ 234. Consultado en el Registro de la Propiedad Mercantil de la Dirección Provincial del Ministerio de Justica en Pinar del Río, 2013

EXtRemerA, D.: Cooperativas de la construcción en Cuba: cinco años después 2018. En http://www.cubadebate.cu/?s=Cooperativas

Fernández, L. (compiladora): Pensando en la personalidad, Tomo I. Editorial Félix Varela La Habana 2005.

Gaceta Oficial No. 53, Extraordinaria, de 11 de diciembre de 2012.

Gargallo, A., Freundich, F.: «Percepciones de los socios y no socios cooperativistas sobre la satisfacción laboral». REVESCO No. 103 (2010) pp. 33-58. En https://revistas.ucm.es/index.php/REVE/article/view/ REVE1010440033A/18585

GómEZ, G.: «Sentirse parte. Un estudio sobre la Percepción Social de la discriminación por VIH y sida». Tesis en opción al grado de Máster en Psicología Social y Comunitaria. Facultad de Psicología. Universidad de La Habana 2009.

González-Guija: La influencia de los medios en la percepción social de la delincuencia ejercida sobre la mujer. En soporte digital. s/f.

HeRnÁNDEZ, O.: «Consideraciones en torno al enfoque de género en las Cooperativas no Agropecuarias en Pinar del Río». Revista Deusto Estudios Cooperativos No. 122019 pp. 141-166. En https://dialnet.unirioja.es/servlet/ articulo?codigo $=6887106$

HeRnÁNDEZ, O.: «La relación género y cooperativa en Cuba. Perspectivas dentro del marco jurídico experimental de la cooperativa». Revista de Estudios Cooperativos, No. 22013 pp. 133-156. En https://dialnet.unirioja.es/servlet/ articulo?codigo $=4191082$

HeRnÁNDEZ, O.: «La relación género y cooperativa en el ordenamiento jurídico cubano. Una aproximación a su realidad y sus perspectivas ante el proceso de perfeccionamiento del modelo económico en el país». Boletín Anual de la Asociación Internacional de Derecho Cooperativo, Deusto, No. 46, 2012 pp. 297-320. En http://baidc.revistas.deusto.es/article/view/140

HernÁndez, O.: "Las cooperativas no agropecuarias en Pinar del Río. Una aproximación al enfoque de género en su funcionamiento». Revista Deusto Estudios Cooperativos No. 62015 pp. 159-179. En https://dialnet.unirioja. es/servlet/articulo?codigo $=5205846$

Hernández, O; VAlle, D.: "La Cooperativa No Agropecuaria Atelier "La Moda": una aproximación a la experiencia de una cooperativa de mujeres». En Congreso Nacional de Estudios del Trabajo: El trabajo en conflicto: dinámicas y expresiones en el contexto actual. Buenos Aires 2017. En https:// www.aset.org.ar/2017/ponencias/11_Hernandez_Aguilar.pdf

IGLESIAS, E., BARCENAS, J.: «Un estudio sobre la percepción social de las cooperativas en Cuba». Cooperativa de servicios contables ACERCO. En Evento Internacional de Psicología «Personas de Miradas Diversas» 2017. 
Universidad de Pinar del Río. En http://eventos.upr.edu.cu/index.php/ psicologia2017PSC/paper/viewFile/1276/828

Morales, D.; RodríGuez, J.; SOSA, D.; TADEO, M.: La percepción social con base a los estereotipos 2016. En http://vinculacion.dgire.unam.mx/vinculacion-1/ Memoria-Congreso-2016/trabajos-ciencias-sociales/sociologia/13.pdf

PADRón, L.; OJeda, L.; OrtiZ, M.: «Desempeño del servicio logístico al cliente. Caso de estudio Cooperativa no Agropecuaria Atelier "La Moda"». Revista Cooperativismo y Desarrol/o 5(2) 2017 pp. 233-240. En http://coodes.upr. edu.cu/index.php/coodes/article/view/181/330

PalaCIO, Y.: Identidad Nacional y Cine Cubano Contemporáneo: una visión desde la subjetividad del espectador. Tesis en opción al grado de Máster en Psicología Social y Comunitaria. Universidad de La Habana, 2005

Perera, M.: Informe Final del proyecto de Investigación Percepción Social del Dirigente: Programa Nacional de Ciencia y Técnica. «El trabajo con los cuadros (trabajo inédito). Centro de Investigaciones Psicológicas y Sociológicas. La Habana, 2002

Periódico Granma: Cooperativas no Agropecuarias. Luces y sombras. En http:// www.granma.cu/cuba/2014-02-07/luces-y-sombras 2014.

PINEEIRO, C.: Cooperativas y socialismo. Una mirada desde Cuba. Editorial Camino, La Habana 2012.

Ponce, F.; PerugorríA, D.; Salgado, R.: Las cooperativas no agropecuarias en la actividad de los servicios técnicos, personales y de uso doméstico en la provincia de Pinar del Río: desarrollo, limitaciones y propuestas para perfeccionar su gestión s/f. En https://dialnet.unirioja.es/descarga/articulo/5294661. pdf

RodríGUez, O. y Rivera, J.: «Historia y realidad jurídica de la cooperativa en Cuba. Aproximación desde la experiencia práctica a las noveles cooperativas no agropecuarias en Pinar del Río». Boletín de la Asociación Internacional de Derecho Cooperativo, 49 (2015) pp. 195-222. En http://baidc. revistas. deusto.es/article/download/757/916

RodríGuez, O.: Presupuestos teóricos para el redimensionamiento constitucional de la Cooperativa en Cuba. Vincere Editora, Brasília-DF, 2017.

SIFONTE, Y.: Cooperativas-Empresas: Los eslabones perdidos de una cadena 2019 En http://www.cubadebate.cu/especiales/2019/04/04/cooperativasempresas-los-eslabones-perdidos-de-una-cadena/\#.XoOMWSXB-EC

Torada, R. y Moreno, N.: Salud laboral y género. Mujer y trabajo. Problemática actual. Editorial Germania, Valencia (s/f) pp. 32-40

VAlLE, D.: Efectos de las exigencias laborales de una jornada de trabajo en la salud de profesores de la Facultad de Ciencias Sociales y Humanísticas de la Universidad de Pinar del Río. Tesis de Licenciatura. Facultad de Psicología, Universidad de La Habana 2014.

VIELICHKOSKY, B., ZINCHENKO, V., LURIA,. A.: «Psicología de la percepción». Editorial Universidad de La Habana 1982 en Bello, Z.: Psicología general. Editorial Félix Varela. La Habana, 2007. 


\section{Derechos de autor}

La revista Deusto Estudios Cooperativos es una revista de acceso abierto lo que significa que es de libre acceso en su integridad inmediatamente después de la publicación de cada número. Se permite su lectura, la búsqueda, descarga, distribución y reutilización legal en cualquier tipo de soporte sólo para fines no comerciales y según lo previsto por la ley; sin la previa autorización de la Editorial (Universidad de Deusto) o el autor, siempre que la obra original sea debidamente citada (número, año, páginas y DOI si procede) y cualquier cambio en el original esté claramente indicado.

\section{Copyright}

The Deusto Journal of Cooperative Studies is an Open Access journal which means that it is free for full and immediate access, reading, search, download, distribution, and lawful reuse in any medium only for non-commercial purposes, without prior permission from the Publisher or the author; provided the original work is properly cited and any changes to the original are clearly indicated. 